\title{
Study on Credit Rating Model of China's listed Companies Based on the Optimal Segmentation Method
}

\author{
Yinan Ning Yunfei Li \\ China West Normal University School of Mathematics and Information, Nanchong \\ 637009, China
}

\begin{abstract}
Listed companies play a crucial role in the development of China's economy. The accurate evaluation of the credit condition of Listed Companies in China is conducive to better management and healthy development. For this problem, first the samples were scored comprehensively by applying projection pursuit method, so ordered samples were obtained which ensured data comprehensive. Then Fisher optimal segmentation method was used to conduct the clustering analysis of ordered sample. Finally, the credit rating model of Listed Companies in china was obtained. A case study of financial statements of listed companies was also performed.
\end{abstract}

Keywords

Projection pursuit method; Ordered samples; Cluster analysis; Credit rating

\section{基于最优分割法的我国上市公司信用评 级模型研究}

\author{
甯懿楠 李云飞 \\ 西华师范大学数学与信息学院, 南充 637009, 中国
}

摘要：上市公司在我国经济的稳健发展中有至关重要的作用, 对我国上市公司信用状况进行 准确的级别评定有助于公司更好的管理和健康发展。针对这一问题，本文首先运用投影寻踪 法对样本进行综合评分，保证了指标数据全面的前提下，基于综合评分形成有序样本，再利 用 Fisher 最优分割法对有序样本进行了聚类分析，最终得到了我国上市公司的信用评级模 型。最后利用上市公司财务报表数据进行实例分析。

关键词：投影寻踪；有序样本；聚类分析；信用评级

我国是市场经济体制，随着我国 1. 引言 市场经济的快速发展，上市公司的良 
好信用成了我国市场经济稳健发展的 重要前提, 而信用评级有助于上市公 司防范信用风险, 准确的信用级别评 定能够大幅度降低事后的管理成本。

信用评级 ${ }^{[1]}$, 又称资信评级, 是对 评级对象偿还债务的能力和意愿进行 综合性的预测与评价, 其目的是为了 显示评级对象信贷违约风险的大小。 在国外, Altman 建立了 $\mathrm{Z}$ 计分模型和 ZETA 评分模型来评价违约风险 ${ }^{[2]}$; J.P 摩根公司提出了信用度量术 ${ }^{[3]}$; Robert Craig West 将因子分析与 logit 估计相 结合, 构建了一个新的风险预警模型 ${ }^{[4]}$; Serpil Canbas 等学者以主成分分析 求因子得分, 再利用判别分析构建了 信用风险预警模型 ${ }^{[5]}$ 。在国内, 丁东洋 等学者基于贝叶斯方法对违约概率和 信用评级进行了研究 ${ }^{[6]}$; 史小康等学者 将两类有偏 logistic 分布应用在信用评 分模型中 ${ }^{[7]}$; 沈霞采用因子分析法和定 性打分法对我国 P2P 网贷平台信用进 行评级分析 ${ }^{[8]}$ 。

在已有的研究中, 信用评级大多 是建立在定性、定量分析的基础上, 采用指标的篎选、权重的确定进行信 用评分, 再进行信用评级。本文旨在 已有研究的基础上, 利用能够有效处 理非线性、非正态数据的投影寻踪法 对样本进行综合评分, 基于综合评分 对样本进行排序, 再利用 Fisher 最优 分割法对有序样本进行了聚类分析, 最终构建一个新的信用评级模型。

\section{2. 决策矩阵规范化处理}

本文旨在对四川33家上市公司 $x_{i}(1 \leq i \leq n), n=33$ 进行研究; 抽取了财 务报表中盈利能力、成长能力、偿还 能力和营运能力四个方面 50 个指标 $u_{j}(1 \leq j \leq m), m=50$, 称 $\mathrm{U}$ 为属性 集; 报表中各指标的具体数值为 $a_{i j}$, 构成决策矩阵 $A=\left(a_{i j}\right)_{n \times m}$ (表 1$)$ :
表 1 决策矩阵 A

\begin{tabular}{c|cccc}
\hline & $u_{1}$ & $u_{2}$ & $\cdots$ & $u_{m}$ \\
\hline$x_{1}$ & $a_{11}$ & $a_{12}$ & & $a_{1 m}$ \\
$x_{2}$ & $a_{21}$ & $a_{22}$ & & $a_{2 m}$ \\
$\cdots$ & $\cdots$ & $\cdots$ & & $\cdots$ \\
$x_{n}$ & $a_{n 1}$ & $a_{n 2}$ & & $a_{n m}$ \\
\hline
\end{tabular}

决策矩阵规范化实质是数据规范 化。数据规范化 ${ }^{[9]}$ 是通过将属性数据按 某一种变换, 将原来的有量纲的值转 换为无量纲的值, 以此来消除量纲间 的影响。由于财务指标有效益型（指 标值越大越好）、成本型（指标值越 小越好) 和固定型（指标值为某个值 最好) 之分, 故按以下变换对指标值 进行预处理:

效益型:

$$
\begin{aligned}
& b_{i j}=\frac{a_{i j}-\min _{1 \leq i \leq n} a_{i j}}{\max _{1 \leq i \leq n} a_{i j}-\min _{1 \leq i \leq n} a_{i j}}, \\
& (1 \leq i \leq n, 1 \leq j \leq m) \quad ;
\end{aligned}
$$

成本型:

$$
\begin{gathered}
b_{i j}=\frac{\max _{1 \leq i \leq n} a_{i j}-a_{i j}}{\max _{1 \leq i \leq n} a_{i j}-\min _{1 \leq i \leq n} a_{i j}}, \\
(1 \leq i \leq n, 1 \leq j \leq m) ;
\end{gathered} ;
$$

固定型:

$$
\begin{aligned}
& b_{i j}=1-\frac{\max _{1 \leq i \leq n} a_{i j}-a_{j}}{\max _{1 \leq i \leq n}\left|a_{i j}-a_{j}\right|}, \\
& (1 \leq i \leq n, 1 \leq j \leq m) \quad ;
\end{aligned}
$$

其中 $\mathrm{n}$ 是研究对象个数, $\mathrm{m}$ 是属性个 数, $b_{i j}$ 是标准化后的数据, 得到标准 化后的判断矩阵 B。

\section{3. 基于投影寻踪法进行样本排序}

\section{1. 投影寻踪法原理}

投影寻踪 ${ }^{[10]}$ 是一种分析与处理高 维观测数据, 特别是针对非正态的高 维数据的新型统计方法, 其基本思路 是将高维的观测数据投影到低维(1-3 
维)的子空间上，从而寻找出能够反映 原数据结构或者特征的投影, 最终达 到降维的目的。

利用投影寻踪方法降维步骤 ${ }^{[11]}$ :

（1）选取线性投影模型。构造维 数为 $\mathrm{m}$ 的单位向量 $\left(h_{1}, h_{2}, \ldots, h_{m}\right)$ 为投影 方向, 其中 $\mathrm{m}$ 是指标的个数, 则研究 对象 $\mathrm{i}$ 在一维线性空间的投影值为 $y_{i}$, $y_{i}$ 的计算公式为:

$$
y_{i}=\sum_{j=1}^{m} h_{j} b_{i j}(1 \leq i \leq n) \quad \text {; }
$$

（2）构造目标函数。在综合投影 值 $y_{i}$ 时, 局部投影点越多且整体上越 分散开能最大程度地提取 $b_{i j}$ 的变异信 息。故构造投影目标函数 $Q(h)=S(h) \bullet D(h)$, 类间距离 $S(h)$ 越 大, 分布越散开; 局部密度 $D(h)$ 越 大，局部越集中。其中

$$
\begin{aligned}
& S(h)=\left[\sum_{i=1}^{n}\left(y_{i}-\overline{y_{h}}\right)^{2} /(n-1)\right]^{\frac{1}{2}}, . \\
& D(h)=\sum_{i=1}^{n} \sum_{j=1}^{n}\left(R-r_{i j}\right) f\left(R-r_{i j}\right), \ldots
\end{aligned}
$$

其中 $\overline{y_{h}}$ 是投影方向上的均值; $r_{i j}=\left|y_{i}-y_{j}\right|(i, j=1,2, \ldots, n)$ 为投影值间 的距离; $\mathrm{R}$ 为局部密度的窗口半径, 一般可取 $0.1 S(h)^{[12] ;} f\left(R-r_{k}\right)$ 为单位 阶跃函数:

$$
f\left(R-r_{i j}\right)=\left\{\begin{array}{l}
1, R \geq r_{i j} \\
0, R<r_{i j}
\end{array}\right.
$$

（3）优化结果。当 $Q(h)$ 取得最大 值时所对应的投影方向就是最佳投影 方向, 即

$$
\left\{\begin{aligned}
\max Q(h) & =S(h) \bullet D(h) \\
s \bullet t\|h\| & =\sum_{j=1}^{m} h_{j}{ }^{2}=1
\end{aligned}\right. \text { 的解。 }
$$

（4）根据优化结果求得一维投影 值 $y_{i}$ 。将求得的 $h^{*}$ 代入(4)式, 求得最 佳投影值 $y_{i}{ }^{*}$, 实现降维。

\section{2. 基于投影寻踪法进行样本排序}

依据最佳投影值 $y_{i}{ }^{*}$, 求得上市公 司的综合评分 $z_{i}, z_{i}=y_{i}^{*}$, 利用 $\mathrm{z}$ 值 的大小进行排序, 得到有序样本 $X_{(i)}(i=1,2, \ldots, n)$ 。

\section{4. 上市公司信用评级模型的建立}

对有序样本 $X_{(1)}, X_{(2)}, \ldots, X_{(n)}$, $\left(X_{(i)}(i=1,2, \ldots, n)\right.$ 为 $\mathrm{m}$ 维向量), 将 $\mathrm{n}$ 个样品分为 $\mathrm{k}$ 类的方法有 $C_{n-1}^{k-1}$ 种, 其 中每 一 种的形式为 $\left\{X_{(i)}, X_{(i+1)}, \ldots, X_{j}\right\},(j>i)$, Fisher 最优 分割法是通过构建一个损失函数, 在 损失函数意义下求得最佳分割[13]。

Fisher 最优分割法具体步骤[14]

(1) 定义类的直径

类 的 直 径 $D(i, j)=\sum_{t=i}^{j}\left(X_{(t)}-\bar{X}_{(G)}\right)^{\prime}\left(X_{(t)}-\bar{X}_{(G)}\right) ，$ 其中 $\bar{X}_{(G)}$ 是某一分类 $\mathrm{G}$ 包含的样本 $\left\{X_{(i)}, X_{(i+1)}, \ldots, X_{j}\right\},(j>i)$ 的均值 向 量, 简记为 $G=\{i, i+1, \ldots, j\}, \bar{X}_{(G)}$ 的 计算公式为 $\bar{X}_{(G)}=\frac{1}{j-i+1} \sum_{t=i}^{j} X_{(t)}$.

(2) 定义分类的损失函数 用 $b(n, k)$ 表示将 $\mathrm{n}$ 个有序样本分 为 $\mathrm{k}$ 类的某一种分类法, 简记为:

$$
\begin{aligned}
& G_{1}=\left\{i_{1}, i_{1}+1, \ldots, i_{2}-1\right\} ; \\
& G_{2}=\left\{i_{2}, i_{2}+1, \ldots, i_{3}-1\right\} ; \\
& \ldots \ldots \ldots \ldots \ldots \ldots \ldots \ldots . . . \ldots \ldots \ldots . . . \\
& G_{k}=\left\{i_{k}, i_{k}+1, \ldots, n\right\},
\end{aligned}
$$

定义上述分类法的损失函数为:

$$
L[b(n, k)]=\sum_{t=1}^{k} D\left(i_{t}, i_{t+1}-1\right),
$$

当 $L[b(n, k)]$ 最小时, 表示各类的离差 平方和最小, 此时的分类最合理 
(3) 寻找最小损失函数

利用以下递推公式寻找最优解:

$\left\{\begin{array}{c}L[b(n, 2)]=\min _{2 \leq j \leq n}\{D(1, j-1), D(j, n)\}, \\ L[b(n, k)]=\min _{k \leq j \leq n}\{L[b(j-1, k-1)]+D(j, n)\end{array}\right.$

$(j=2,3, \ldots, n)$

(4) 最优解的求解

第一步: 寻找分点 $j_{k}, s \bullet t$

$L[b(n, k)]=\min _{k \leq j \leq n}\{L[b(j-1, k-1)]+D(j, n)\}$

最小, 求得第 $\mathrm{k}$ 类 $G_{k}=\left\{i_{k}, i_{k}+1, \ldots, n\right\}$,

第二步: 找到分点 $j_{k-1}$, 重复进行 第一步，直至找到所有分类。

\section{5. 实例分析}

\section{1. 研究结果分析}

本文以四川上市公司为对象，篮 选在年度财务报表中各指标数据齐全 的 33 家上市公司 1 进行研究。

通过决策矩阵规范化处理, 利用 matlab 求得当投影指标值 $Q(h)$ 取得最 大值 3.0154 时所对应的投影方向 $h^{*}=$ ( 0.14086,0.14575,0.079595,0.17445,0.1 $8101,0.1297,0.1476,0.10612,0.14967,0.1$ $1977,0.18651,0.071687,0.18096,0.05287$ $4,0.09253,0.15847,0.060744,0.0531,0.07$ $0938,0.11245,0.032076,0.22141,0.15063$, $0.18117,0.13903,0.10689,0.18697,0.1320$ $8,0.22163,0.21832,0.18353,0.072344,0.1$ $1779,0.17004,0.12696,0.0068273,0.1321$ $6,0.11477,0.015865,0.033972,0.079568,0$. $11159,0.20622,0.10809,0.22378,0.10723$, $0.17399,0.19473,0.09205,0.20684)$ ，将 $h^{*}$ 代入(1)式, 求得最佳投影值 $y_{i}^{*}$, 最 终得到四川 33 个上市公司得分及排名 (表 2), 并通过 $y_{i}{ }^{*}$ 的大小关系生成有序 样本 $z_{(i)}$ 。

1 以四川 2015-2016年企业财务报表中无缺失数 据的 33 家上市公司为例, 数据源:

http://money.163.com/.
表 2 上市公司得分及排名

\begin{tabular}{|c|c|c|c|}
\hline 公司名称 & $\begin{array}{c}\text { 大通燃 } \\
\text { 气 }\end{array}$ & $\begin{array}{c}\text { 四川九 } \\
\text { 洲 }\end{array}$ & $\begin{array}{c}\text { 创维数 } \\
\text { 字 }\end{array}$ \\
\hline 得分/排名 & $\begin{array}{c}4.07647 \\
1 / 4 \\
\end{array}$ & $\begin{array}{c}3.65899 \\
5 / 15 \\
\end{array}$ & $\begin{array}{c}3.55557 \\
2 / 18 \\
\end{array}$ \\
\hline 公司名称 & 新希望 & $\begin{array}{c}\text { 海特高 } \\
\text { 新 }\end{array}$ & $\begin{array}{c}\text { 富临运 } \\
\text { 业 }\end{array}$ \\
\hline 得分/排名 & $\begin{array}{c}4.10253 \\
4 / 3\end{array}$ & $\begin{array}{c}3.92510 \\
3 / 6\end{array}$ & $\begin{array}{c}3.84082 \\
8 / 8\end{array}$ \\
\hline 公司名称 & $\begin{array}{c}\text { 台海核 } \\
\text { 电 }\end{array}$ & $\begin{array}{c}\text { 天齐锂 } \\
\text { 业 }\end{array}$ & $\begin{array}{l}\text { 云图控 } \\
\text { 股 }\end{array}$ \\
\hline 得分/排名 & $\begin{array}{c}3.41897 \\
8 / 23\end{array}$ & $\begin{array}{c}3.87837 \\
8 / 7\end{array}$ & $\begin{array}{c}3.52440 \\
2 / 19\end{array}$ \\
\hline 公司名称 & $\begin{array}{c}\text { 成都路 } \\
\text { 桥 }\end{array}$ & $\begin{array}{c}\text { 天翔环 } \\
\text { 境 }\end{array}$ & $\begin{array}{c}\text { 创意信 } \\
\text { 息 }\end{array}$ \\
\hline 得分/排名 & $\begin{array}{c}3.18383 \\
6 / 27\end{array}$ & $\begin{array}{c}3.46598 \\
5 / 21\end{array}$ & $\begin{array}{c}3.69302 \\
7 / 13\end{array}$ \\
\hline 公司名称 & $\begin{array}{c}\text { 迈克生 } \\
\text { 物 }\end{array}$ & $\begin{array}{c}\text { 四川路 } \\
\text { 桥 }\end{array}$ & $\begin{array}{c}\text { 易见股 } \\
\text { 份 }\end{array}$ \\
\hline 得分/排名 & $\begin{array}{c}4.33734 \\
7 / 2\end{array}$ & $\begin{array}{c}3.12166 \\
6 / 29\end{array}$ & $\begin{array}{c}3.40349 \\
7 / 24\end{array}$ \\
\hline 公司名称 & $\begin{array}{c}\text { 岷江水 } \\
\text { 电 }\end{array}$ & $\begin{array}{c}\text { 成发科 } \\
\text { 技 }\end{array}$ & $\begin{array}{c}\text { 通威股 } \\
\text { 份 }\end{array}$ \\
\hline 得分/排名 & $\begin{array}{c}3.60286 \\
5 / 17\end{array}$ & $\begin{array}{c}3.38826 \\
3 / 25\end{array}$ & $\begin{array}{c}3.95582 \\
8 / 5\end{array}$ \\
\hline 公司名称 & $\begin{array}{c}\text { 蓝光发 } \\
\text { 展 }\end{array}$ & $\begin{array}{c}\text { 西昌电 } \\
\text { 力 }\end{array}$ & 大西洋 \\
\hline 得分/排名 & $\begin{array}{c}3.71526 \\
3 / 11\end{array}$ & $\begin{array}{c}3.81573 / \\
9\end{array}$ & $\begin{array}{c}3.81125 \\
8 / 10\end{array}$ \\
\hline 公司名称 & $\begin{array}{c}\text { 川投能 } \\
\text { 源 }\end{array}$ & $\begin{array}{c}\text { 宜宾纸 } \\
\text { 业 }\end{array}$ & $\begin{array}{c}\text { 茂业商 } \\
\text { 业 }\end{array}$ \\
\hline 得分/排名 & $\begin{array}{c}4.53029 \\
3 / 1\end{array}$ & $\begin{array}{c}1.43198 \\
6 / 33\end{array}$ & $\begin{array}{c}3.67963 \\
6 / 14\end{array}$ \\
\hline 公司名称 & $\begin{array}{c}\text { 东方电 } \\
\text { 气 }\end{array}$ & $\begin{array}{c}\text { 四川成 } \\
\text { 渝 }\end{array}$ & $\begin{array}{c}\text { 东材科 } \\
\text { 技 }\end{array}$ \\
\hline 得分/排名 & $\begin{array}{c}3.49202 \\
5 / 20\end{array}$ & $\begin{array}{c}3.21804 \\
6 / 26\end{array}$ & $\begin{array}{c}3.70937 \\
7 / 12\end{array}$ \\
\hline 公司名称 & $\begin{array}{c}\text { 和邦生 } \\
\text { 物 }\end{array}$ & $\begin{array}{c}* \mathrm{ST} \text { 三 } \\
\text { 泰 }\end{array}$ & $\begin{array}{c}* \text { ST 天 } \\
\text { 仪 }\end{array}$ \\
\hline 得分/排名 & $\begin{array}{c}3.60376 \\
4 / 16\end{array}$ & $\begin{array}{c}3.42402 \\
8 / 22\end{array}$ & $\begin{array}{c}2.77875 \\
3 / 31\end{array}$ \\
\hline 公司名称 & $\begin{array}{c}* \mathrm{ST} \text { 钒 } \\
\text { 钛 }\end{array}$ & $\begin{array}{c}* \text { *ST 华 } \\
\text { 泽 }\end{array}$ & $\begin{array}{c}* \text { *ST 金 } \\
\text { 宇 }\end{array}$ \\
\hline 得分/排名 & $\begin{array}{c}2.77824 \\
8 / 32\end{array}$ & $\begin{array}{c}3.17579 \\
7 / 28\end{array}$ & $\begin{array}{c}2.77898 / \\
30\end{array}$ \\
\hline
\end{tabular}

对有序样本 $z_{(i)}, i=1,2, \ldots, 33$ 进行 Fisher 最优分割, 得到不同分类数 $\mathrm{k}$ 的 最小损失函数图(图 1): 


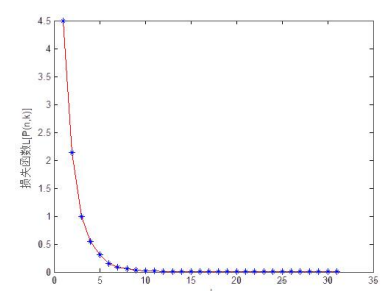

图 1 最小损失函数随分类数 $\mathrm{K}$ 的变化曲线

由图 1 可以看出, 图像在 $\mathrm{k}=4-6$ 处有明显转折, 根据我国信用风险四 分法的需要, 故本文选择将上市公司 信用等级分成 4 类: Prime-1(P-1): 偿 付能力最强, 信用最好; Prime-2(P$2)$ : 偿付能力较强, 信用较好; Prime$3(\mathrm{P}-3)$ : 偿付能力尚可接受, 信用一 般; Not Prime：不在任何 Prime 评级 之内, 信用很差 ${ }^{[15]}$ 。

根据 Fisher 最优分割找最优解的 步骤, 根据 matlab 求解最小损失函数 可得:

$L[P(33,4)]=0.5493(30)$, $L[P(29,3)]=0.5493(19)$, $L[P(18,2)]=0.3433(5)$, 所以得到最终 4 类分类 $G_{i}(i=1,2, \ldots, 4)$ :

$G_{1}=\left\{z_{1}, \ldots, z_{4}\right\} ， G_{2}=\left\{z_{5}, \ldots, z_{18}\right\}$, $G_{3}=\left\{z_{19}, \ldots, z_{29}\right\} ， G_{4}=\left\{z_{30}, \ldots, z_{33}\right\}$, 从而得到 33 个上市公司的信用评级结 果（见表 3) :

表 3 信用评级结果

\begin{tabular}{c|c|c}
\hline 信用综合得分区间 & 信用等级 & $\begin{array}{c}\text { 违约样本 } \\
\text { 所占比例 }\end{array}$ \\
\hline$(3.955828,+\infty)$ & P-1 & $0.00 \%$ \\
\hline$(3.524402,3.955828]$ & P-2 & $0.00 \%$ \\
\hline$(2.77898,3.524402]$ & P-3 & $15.38 \%$ \\
\hline$(-\infty, 2.77898]$ & Not Prime & $100 \%$ \\
\hline
\end{tabular}

根据信用风险四分法可知, 从 P-1 级到 Not Prime 级出现信用危机的概率 是逐渐增大的, 由表 3 结果可以看 出, 从 P-1 级到 Not Prime 级违约公司 所占的比例也逐渐增大, 通过表 2、3
可以看出, 本文样本中的 ST 公司也完 全划分到了 P-3、 Not Prime 级中, 而 且 Not Prime 级 $\left(G_{4}\right)$ 中的样本公司完全 是 ST 公司, 由此可以看出本文方法对 上市公司进行信用评级不仅可以得到 与实情相符的评级结果, 而且可以得 到各个信用等级的得分区间, 通过得 分区间的划分可以判断某个新的上市 公司的信用等级。

\section{2. 应用分析}

本文考虑到地域差异对财务指标 的影响, 故选择川渝两地的上市公司 进行对比研究, 以四川地区的上市公 司作为研究对象, 将研究结果应用到 重庆地区上市公司中, 以此来验证本 文研究结果。

首先, 对重庆数据齐全的 14 家上 市公司的各财务数据 ${ }^{2}$ 进行规范化处 理, 利用 4.1 中所求得的最佳投影方向 $h^{*}$, 得到重庆上市公司信用综合得分 及排名 (表 4):

表 4 重庆上市公司得分及排名

\begin{tabular}{c|c|c|c}
\hline 公司名称 & $\begin{array}{c}\text { 重庆港 } \\
\text { 九 }\end{array}$ & $\begin{array}{c}\text { 重庆路 } \\
\text { 桥 }\end{array}$ & $\begin{array}{c}\text { 力帆股 } \\
\text { 份 }\end{array}$ \\
\hline 得分/排名 & $\begin{array}{c}3.60288 / \\
9\end{array}$ & $\begin{array}{c}3.68114 \\
9 / 7\end{array}$ & $\begin{array}{c}3.27968 \\
3 / 12\end{array}$ \\
\hline 公司名称 & $\begin{array}{c}\text { 蓝黛传 } \\
\text { 动 }\end{array}$ & $\begin{array}{c}\text { 川仪股 } \\
\text { 份 }\end{array}$ & $\begin{array}{c}\text { 迪马股 } \\
\text { 份 }\end{array}$ \\
\hline 得分/排名 & $\begin{array}{c}3.76096 / \\
6\end{array}$ & $\begin{array}{c}3.58757 / \\
10\end{array}$ & $\begin{array}{c}3.10775 \\
6 / 14\end{array}$ \\
\hline 公司名称 & $\begin{array}{c}\text { 金科股 } \\
\text { 份 }\end{array}$ & $\begin{array}{c}\text { 奥瑞德 } \\
\text { 申宗动 } \\
\text { 力 }\end{array}$ \\
\hline 得分/排名 & $\begin{array}{c}3.15200 \\
8 / 14\end{array}$ & $\begin{array}{c}3.78445 \\
7 / 5\end{array}$ & $\begin{array}{c}3.95359 \\
6 / 3\end{array}$ \\
\hline 公司名称 & $\begin{array}{c}\text { 华邦健 } \\
\text { 康 }\end{array}$ & $\begin{array}{c}\text { 重庆燃 } \\
\text { 气 博腾股 } \\
\text { 份 }\end{array}$ \\
\hline 得分/排名 & $\begin{array}{c}3.60465 \\
3 / 8\end{array}$ & $\begin{array}{c}3.83646 / \\
4\end{array}$ & $\begin{array}{c}3.56827 \\
3 / 11\end{array}$ \\
\hline 公司名称 & 重庆水 & 隆金通 & \\
\hline 得分/排名 & $\begin{array}{c}\text { 务 } \\
\text { 用 }\end{array}$ & \\
\hline
\end{tabular}

2 以重庆2015-2016年的企业财务报表中无缺失 数据的14家上市公司为例, 数据源: http://money.163.com/. 
其次, 根据表 3 中信用等级与信 用得分区间的一一对应关系，得到重 庆地区上市公司信用评级结果 (表 5):

表 5 信用评级结果

\begin{tabular}{c|c}
\hline 信用等级 $/$ 个数 & 公司名称 \\
\hline P-1 级 $/ 2$ 个 & 重庆水务, 隆金通用 \\
\hline P-2 级 $/ 9$ 个 & $\begin{array}{l}\text { 重庆港九, 重庆路桥, 蓝 } \\
\text { 黛传动, 川仪股份, 奥瑞 } \\
\text { 德, 申宗动力, 华邦健 } \\
\text { 康, 重庆燃气, 博腾股份 }\end{array}$ \\
\hline P-3 级 $/ 3$ 个 & $\begin{array}{c}\text { 力帆股份, 迪马股份, 金 } \\
\text { 科股份 }\end{array}$ \\
\hline Not Prime 级 $/ 0$ 个 & 无 \\
\hline
\end{tabular}

从表 5 可以看出, 在重庆地区的 评级结果中, P-1 级 2 个, P-2 级 9 个, P-3 级 3 个, Not Prime 级 0 个, 处于 P-2 级的公司较多, 重庆地区 14 个上 市公司中没有 ST 公司, Not Prime 级 中也没有 ST 公司, 结果与实际相符。

\section{6. 结语}

本文运用投影寻踪法对样本进行 综合评分, 基于综合评分形成有序样 本, 再利用Fisher最优分割法对有序 样本进行了聚类分析, 为上市公司信 用评级提供了新的方法。

\section{致谢}

感谢四川省教育厅自然科学重点 项目（13ZA0016）; 西华师范大学博 士科研启动基金（12B025）; 西华师 范大学英才科研基金项目（17YC381） 的资助。

\section{参考文献}

[1] 谢多, 冯光华编著. 信用评级 [M]. 北京. 中国金融出版社, 2014 .

[2] E.I. Altman, P.R. Haldeman, P. Narayanan. ZETA Analysis: A New Model to Identify Bankruptcy Risk of Corporations[J]. Journal of Banking and Finance, 1997(1): 2954.

[3] J.P. Morgan. Creditmetrics
Technical Document[C]. New York, April 2, 1997.

[4] Robert Craig West. A factoranalytic Approach to Bank Condition[J]. Journal of Banking \& Finance, 1985(2).

[5] Serpil Canbas, Altan Cabuk, Suleyman Bilgin Kilic. Prediction of Commercial Bank Failure Via multivariate Statistical Analysis of Financial Structures: The Turkish Case[J]. European Journal of Operational Research, 2005(2).

[6] 丁东洋, 周丽莉. 基于贝叶斯方 法的信用评级模型构建与违约概 率估计 $[\mathrm{J}]$. 统计与信息论坛, 2010 (第9期).

[7]史小康, 常志勇. 两类有偏 logistic 分布在信用评分模型中的 应用 $[\mathrm{J}]$. 统计与决策, 2015 (第 14 期)：19-23.

[8] 沈霞. 我国P2P网贷平台信用评级 研究 $[\mathrm{J}]$. 西部金融，2017(第1期)： 66-69.

[9] 李美娟, 陈国宏, 陈衍泰。综合 评价中指标标准化方法研究 [J]. 中国管理科学, 2004,12(z1): 45-48.

[10] 万中英，王明文，揭安全，万剑 怡. 投影寻踪模型中投影指标的 改进 $[\mathrm{J}]$. 江西师范大学学报 (自然 科学版), 2013 (第3 期).

[11] 田铮. 投影寻踪方法与应用 [M]. 西安. 西北工业大学出版社, 2008.

[12] 王硕，张礼兵，金菊良. 系统预 测与综合评价方法 $[\mathrm{M}]$. 合肥. 合 肥工业大学出版社, 2006.

[13] 严广松, 路允芳. 多维有序样本 的聚类方法研究 $[\mathrm{J}]$. 统计与决策, 2008 (第4期)：29-31.

[14] 高惠璇. 应用多元统计分析 [M]. 北京. 北京大学出版社, 2005.

[15] 黄献. 评级阴谋-看透经济危机真 相的第一本书 $[\mathrm{M}]$. 北京: 中国友 谊出版公司, 2013. 